\title{
RF Induced Temperature Monitoring in Cochlear Implant Electrodes
}

\author{
Lisa Obmascher \\ Institute for Ion Physics and Applied Physics \\ Technikerstr. 25/3, Innsbruck, Austria \\ lisa.obmascher@student.uibk.ac.at
}

\section{Extended Abstract}

Although magnetic resonance imaging (MRI) is generally regarded as a safe and non-invasive diagnostic method, its combined electromagnetic fields result in potential hazards for patients with implanted medical devices, such as cochlear implants. For example, the electrically conducting components of the medical implant interact with the strong electromagnetic fields in the MRI Scanner, which can result in heating of the surrounding tissue [1]. In order to avoid harm to the patient, the radio frequency (RF) induced temperature rise must be minimized. As a result, quantitative information about the temperature rise is crucial.

Existing temperature measurement techniques require complex setups with external sensors, which have to be positioned very precisely and in immediate proximity to the implant inside the MRI scanner. This could affect the reproducibility and accuracy of the measurement - also by introducing additional disturbances to the local RF field. As a consequence, accompanying time and cost intensive numerical simulations of the temperature change in virtual bodies exposed to MRI RF fields are required in order to assess the implant safety in real life settings [2].

A prototype for the quantification of RF induced temperature increase in a cochlear implant (MED-EL Elektromedizinische Geräte $\mathrm{GmbH}$, Austria) electrode tip was realized, which can be fully integrated into the existing implant housing of $\mathrm{LxWxH}=(2 \times 1 \times 0.6) \mathrm{cm}$ and electrode of diameter $0.5 \mathrm{~mm}$. Thus, the interference between the RF field and the measurement system itself is negligible and the consistent positioning of the sensor leads to high accuracy and reproducibility of the measurements.

For this purpose, a type $\mathrm{T}$ thermocouple $\left(\mathrm{Cu}-\mathrm{CuNi}\right.$, accuracy $\left.0.5^{\circ} \mathrm{C}\right)$ was integrated into a standard $\mathrm{CI}$ electrode, with the sensor tip glued to the outer most electrode contact. The output of the thermocouple is transferred onto a specially designed printed circuit board, which is located inside the cochlear implant housing.

The PCB includes two high frequency filtering stages $(1.6 \mathrm{kHz}$ and $1.6 \mathrm{~Hz})$, which block the induced radiofrequency and only let the DC thermocouple output pass. Consequently, the DC signal is amplified, A/D converted (16 bit resolution, sampling rate $1 \mathrm{~Hz}$ ) and stored on a microchip, where it can be read out via serial communication later on. Two Reed switches are used in order to switch the PCB power supply on and start the data acquisition whenever the cochlear implant is moved into the magnetic field of an MRI scanner.

Functionality and robustness tests were conducted successfully during a standard clinical Turbo Spin Echo sequence in a 3 T MRI scanner (Siemens MAGNETOM Skyra). Future work will focus on combining the temperature monitoring system with a wireless data transmission module, with the aim of enabling direct, real-time communication between the implant and the MRI scanner and/or physician. This could further improve the patient safety in in-vivo settings, by giving an alarm or even triggering an emergency stop of the scan, if the measured temperature exceeds a harmful limit.

\section{References}

[1] H. Bassen, "MRI-induced heating of selected thin wire metallic implants-- laboratory and computational studies findings and new questions raised," Minim Invasive Ther Allied Technol., vol. 15, no. 2, pp. 76-84, 2006.

[2] C. D. Smith, "Interactions of magnetic resonance imaging radio frequency magnetic fields with elongated medical implants," J. Appl. Phys., vol. 87, no. 9, pp. 6188-6190, 2000. 